\title{
Safety of pertussis vaccination in pregnant women in UK: observational study
}

\author{
(c) $\frac{108}{94}$ OPEN ACCESS
}

\section{Katherine Donegan pharmacoepidemiologist, Bridget King scientific assessor, Phil Bryan scientific assessor}

Vigilance and Risk Management of Medicines, Medicines and Healthcare products Regulatory Agency, London SW1W 9SZ, UK

\begin{abstract}
Objective To examine the safety of pertussis vaccination in pregnancy.

Design Observational cohort study.

Setting The UK Clinical Practice Research Datalink.

Participants 20074 pregnant women with a median age of 30 who received the pertussis vaccine and a matched historical unvaccinated control group.
\end{abstract}

Main outcome measure Adverse events identified from clinical diagnoses during pregnancy, with additional data from the matched child record identified through mother-child linkage. The primary event of interest was stillbirth (intrauterine death after 24 weeks' gestation).

Results There was no evidence of an increased risk of stillbirth in the 14 days immediately after vaccination (incidence rate ratio $0.69,95 \%$ confidence interval 0.23 to 1.62$)$ or later in pregnancy $(0.85,0.44$ to 1.61) compared with historical national rates. Compared with a matched historical cohort of unvaccinated pregnant women, there was no evidence that vaccination accelerated the time to delivery (hazard ratio 1.00, 0.97 to 1.02). Furthermore, there was no evidence of an increased risk of stillbirth, maternal or neonatal death, pre-eclampsia or eclampsia, haemorrhage, fetal distress, uterine rupture, placenta or vasa praevia, caesarean delivery, low birth weight, or neonatal renal failure, all serious events that can occur naturally in pregnancy.

Conclusion In women given pertussis vaccination in the third trimester, there is no evidence of an increased risk of any of an extensive predefined list of adverse events related to pregnancy. In particular, there was no evidence of an increased risk of stillbirth. Given the recent increases in the rate of pertussis infection and morbidity and mortality in neonates, these early data provide initial evidence for evaluating the safety of the vaccine in pregnancy for health professionals and the public and can help to inform vaccination policy making.

\section{Introduction}

Pertussis (whooping cough) is a highly contagious disease caused by the bacterium Bordetella pertussis. Symptoms can initially be mild, but serious and fatal complications can occur, particularly in children aged under 3 months. ${ }^{1}$ In the United Kingdom, a primary course of pertussis vaccination is recommended from the age of 2 months. ${ }^{2}$ Uptake for the infant vaccine, and for the additional preschool booster, is high, ${ }^{3}$ and in recent decades pertussis has been well controlled. As elsewhere, however, children remain vulnerable before their first vaccination series.

There are cyclical peaks in rates of pertussis infection, and a sharp increase in confirmed cases was observed in the UK towards the end of 2011. ${ }^{4}$ This increase continued in 2012, with a particularly high rate in infants under 3 months and an increase in infant deaths related to pertussis. This led the UK's Joint Committee on Vaccination and Immunisation to recommend the introduction of a temporary vaccination programme targeting pregnant women at between 28 and 38 weeks' gestation, the aim being to protect children against pertussis before they reach their first routine immunisation. ${ }^{5}$ This programme was introduced uniformly across all four countries in the UK.

Though there are several known minor side effects with pertussis vaccine, it is generally well tolerated in both children and adults. ${ }^{67}$ The programme began on 1 October 2012 with Repevax (Sanofi Pasteur MSD, Berkshire), a combined low dose diphtheria, acellular pertussis, and inactivated poliomyelitis vaccine. As with most vaccines, during clinical development Repevax was not evaluated for use in pregnancy. A similar vaccine, without the inactivated poliomyelitis component, has been routinely recommended during pregnancy in the United States since 2011, with some evidence of safety ${ }^{8-10}$; and the potential benefits ${ }^{11}$ in reducing neonatal morbidity and mortality were expected to outweigh any theoretical risks. Nonetheless, it was essential that plans for active safety surveillance were implemented to rapidly identify potential risks.

In line with its statutory responsibility for vaccine safety, the Medicines and Healthcare products Regulatory Agency undertook a tailored proactive approach to pharmacovigilance, starting as soon as the vaccination campaign began, using electronic medical record data on a monthly basis to identify a large number of vaccinated women in as real time a manner as possible. 
We describe the growing identified cohort, after the first six months of the programme, and present comparative analyses investigating the risk of a range of predefined events. To our knowledge, this is the first large epidemiological study on the safety of pertussis vaccination in pregnancy.

\section{Methods \\ Clinical Practice Research Datalink}

The Clinical Practice Research Datalink (CPRD) collates demographic, clinical, prescribing, test, and referral data extracted from over 650 primary care general practice databases for more than 12.5 million patients throughout the UK (www. cprd.com/intro.asp) with data from the past month for over 3.5 million. The database is geographically representative, and the age and sex distributions of the patients included are similar to those of the population. ${ }^{12}$ GPs receive training on the recording and coding of medical data, which they input as part of their routine clinical practice. Diagnoses, test results, and referrals are recorded as they are made or known by the GP using Read codes. Read codes are a coded thesaurus of clinical terms and are the basic means by which GPs record patient data in electronic medical records in the UK. Adverse events in pregnancy in women presenting to secondary or specialist care should be reported back to the GP and subsequently added to the records, although there can be delays in recording. The CPRD research group assesses the quality of the data to ensure a standard suitable for epidemiological research. The data have been extensively used in observational research, including studies of vaccines ${ }^{13}$ and drugs in pregnancy.

As the pertussis vaccine was principally administered in primary care in general practices to women in their third trimester, use of the CPRD for identifying vaccinated pregnant women was particularly appropriate as data on both pregnancy and vaccination could be rapidly obtained. New data were reviewed on a monthly basis with reassuring interim results available just three months into the campaign. ${ }^{14} \mathrm{We}$ have presented data to 31 March 2013, covering the first six months of the campaign.

\section{Identifying pregnant women, vaccine exposure, and adverse events related to pregnancy}

Pregnant women were identified through Read codes. When identification of the full pregnancy period was required, we used the validated algorithm of Devine and colleagues ${ }^{15}$ to identify pregnancy outcomes, with gestational age estimated from last menstrual period, estimated due dates, or dates of ultrasongraphy when available. Exposure was defined as a record of immunisation during pregnancy with any vaccine containing pertussis on or after 1 October 2012.

Adverse events were identified by using records of clinical diagnoses during pregnancy, with additional data from the matched child record identified through the mother-child linkage compiled by CPRD research staff. ${ }^{16}$ The primary event of interest was stillbirth (intrauterine death after 24 weeks' gestation). Further predefined events of interest included maternal and neonatal death (death within 28 days of delivery), pre-eclampsia and eclampsia, antepartum and postpartum haemorrhage, fetal distress, uterine rupture, placenta praevia, vasa praevia, caesarean delivery, low birth weight (including birth weight $<2500 \mathrm{~g}$ ), and neonatal renal failure. These were chosen as they are all events occurring naturally in the third trimester of pregnancy that could be potentially spontaneously reported as adverse events. We also examined time to delivery.

\section{Statistical analysis}

We took several approaches to the analysis. Two analyses looked at event rates in the CPRD vaccinated cohort compared with published national data. The first focused on the short term risk of adverse events occurring within 14 days of vaccination to examine acute risks and to maximise power by including as many women as possible. To be eligible for this analysis, women had to be aged $\geq 12$, have a record for a vaccination containing pertussis during pregnancy, and have at least 28 days of follow-up data after vaccination to allow adequate time for the recording of adverse events in the GP database. We compared the number of stillbirths in the 14 days after vaccination with the expected figure using observed versus expected methods (exact Poisson confidence intervals) with the background rate, accounting for gestational age, taken from 2010 Office for National Statistics data (ONS) covering England and Wales. ${ }^{17}$ A sensitivity analysis including records of miscarriage (that is, intrauterine death within the first 24 weeks' gestation) after vaccination was also conducted. The numbers of deliveries (live and stillbirths) and neonatal deaths, again in the 14 days after vaccination, were also compared with ONS background rates. ${ }^{17} 18$ The numbers of incident occurrences of the other events of interest were examined.

The second analysis considered events after vaccination across the whole pregnancy. We included only women with a recorded pregnancy outcome and estimated gestational age with follow-up of at least 44 weeks after the date of the last menstrual period to ensure confidence in the available data for the identified population. An estimate for gestational age was vital as the risk of many of the events of interest, in particular stillbirth, changes considerably with gestational age therefore we included only women with a recorded date of last menstrual period or an estimated delivery date from which it could be estimated. The restriction on minimum follow-up was used to try to reduce possible biases caused by differential recording delays for stillbirths and live births. The number of stillbirths was again compared with the expected number based on ONS published data. ${ }^{17}$

Thirdly, we carried out a matched cohort analysis to adjust for two of the most important known risk factors for stillbirth and other adverse events related to pregnancy-namely, maternal and gestational age. A historical unvaccinated cohort was identified from the CPRD that included women aged $\geq 12$ with a recorded pregnancy outcome during 1 October 2010 to 30 September 2012 and no record of a vaccine containing pertussis during or after pregnancy. Each vaccinated woman identified in the second analysis was matched by maternal age ( \pm 2 years) and calendar month of last menstrual period ( \pm 2 months) to up to three unvaccinated historical controls. The index date was the vaccination date or, for the unexposed women, the date they reached the same gestational age as the matched vaccinated woman at the point of their vaccination. The time to delivery was then compared across the vaccinated and unvaccinated cohorts by using a stratified Cox proportional hazards model. To compare the incidence of the events of interest across the whole pregnancy, where the background risk is associated with gestational age, we further refined the matching process to also match by gestational age in weeks at outcome to ensure comparable time at risk. The rate of each adverse event of interest was compared with conditional Poisson regression and birth weight by using the Wilcoxon rank sum test.

We carried out a final matched cohort analysis including all women with a pregnancy outcome during the campaign, regardless of vaccination status, matched to historical controls 
as in the main study but with the index date being the start of the third trimester.

\section{Results}

We identified 20074 pregnant women with a median age of 30 (interquartile range 26-34) with pertussis vaccination during the first six months of the campaign.

\section{Short term risk of adverse events}

In total, $17560(87 \%)$ vaccinated pregnant women had $\geq 28$ days' follow-up data after their vaccination record. They had a median age of 30 (26-34). Gestational age could be estimated for $13371(76 \%)$; the median gestation at vaccination was 31 weeks (29-35). Five had a recorded stillbirth within two weeks of vaccination. From the ONS data on the rate of live births and stillbirths by gestational age, and using the distribution of gestational age at vaccination estimated from the CPRD, we would expect 7.2 stillbirths in this time frame. Therefore, the observed versus expected incidence rate ratio is $0.69(95 \%$ confidence interval 0.23 to 1.62 ), meaning that there is no signal of a short term increased risk of stillbirth after vaccination. As part of a sensitivity analysis, we identified an additional two women with miscarriage after vaccination. In both of these cases, vaccination seemed to have been in the second trimester, when miscarriage rates are considerably higher. The observed versus expected ratio including these two women is 0.97 ( 0.39 to 2.00$)$.

In total there were 1135 pregnancy outcomes (live and stillbirths) within two weeks of vaccination compared with an expected 1115. Therefore, the observed versus expected incidence ratio is 1.02 (0.96 to 1.08), indicating no signal of an increase in the number of pregnancy outcomes within two weeks of vaccination. There were no recorded cases of threatened labour without delivery in the two weeks after vaccination.

There was one recorded incident of pre-eclampsia within two weeks of vaccination. In three women pre-eclampsia at delivery within two weeks of vaccination was recorded; in each case delivery resulted in a live birth. There was just one case of eclampsia after delivery. These figures are reasonably in line with external estimates that suggest a rate of five cases of severe pre-eclampsia per 1000 pregnancies $^{19}$ (although severity is not well recorded in the CPRD so comparison is difficult) and one case of eclampsia per 2000 pregnancies. ${ }^{20}$

In addition, there were three recorded incident cases of antepartum haemorrhage, one of placenta praevia, and one of fetal distress within 14 days of vaccination. There were no recorded cases of uterine rupture, placental abruption, or vasa praevia in the same timeframe.

\section{Time to delivery}

We identified 6185 vaccinated women with adequate follow-up ( $\geq 44$ weeks after estimated date of last menstrual period) and data on pregnancy outcome and gestational age. They had a median age of 30 (26-34) and were vaccinated at a median 33 weeks' gestation (30-36). Each of these women was matched by age and calendar month of last menstrual period to up to three historical unvaccinated controls, who were still pregnant at the matched gestational age at vaccination. There was no significant difference in the time to delivery in the vaccinated and unvaccinated cohorts (median gestation 40 weeks; hazard ratio $1.00,95 \%$ confidence interval 0.97 to 1.02 ).

\section{Overall risk of adverse events of interest}

There were 12 recorded instances of stillbirth after vaccination (table $\Downarrow:$ 12/6185 (0.19\%), about 1 per 500 deliveries). Given the distribution of gestational age at vaccination and the ONS background data on stillbirth rates, under the assumption of no increased risk, 15.8 stillbirths would have been expected in this cohort. The observed versus expected rate ratio is therefore 0.85 (95\% confidence interval 0.44 to 1.61 ). The vaccinated women were further matched to 18496 unvaccinated historical controls as described and the resulting conditional rate ratio for the overall risk of stillbirth in vaccinated versus unvaccinated women was 0.85 ( 0.45 to 1.61$)$.

There were two cases of neonatal death (table $\downarrow$ ) within a week after delivery in addition to the 12 cases of stillbirth. The ONS data suggest the rate of neonatal death is three per 1000 live births. The data have limited power to examine the comparative rate of neonatal death (that is, death within 28 days). A comparison of death within seven days of delivery, however, leads to a rate ratio of 1.00 ( 0.20 to 4.95$)$.

We examined all further prespecified adverse events (table $\Downarrow$ ). There were no significant differences in the rates of any of the pre-specified events, generating no safety signals. There were no records of maternal death, antepartum haemorrhage, uterine rupture, placental abruption, vasa praevia, fetal distress, or child renal failure after vaccination. The median birth weight in the vaccinated cohort was $3500 \mathrm{~g}$ (interquartile range 3100-3800

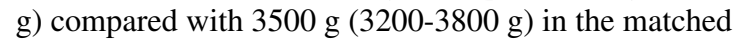
unvaccinated cohort $(\mathrm{P}=0.81)$.

The further sensitivity analysis including all women with a pregnancy outcome during the campaign $(n=9735)$, regardless of vaccination status ( $36 \%$ with no vaccination record) found no significant increases in the risk of any of the predefined adverse events related to pregnancy before and after the introduction of the vaccination campaign, with confidence intervals of a comparable magnitude with the main analyses presented (table $\downarrow$ ). In addition to the events described in the table, single incident cases of antepartum haemorrhage and placental abruption were identified in women eligible for vaccination, while there were no records of maternal death, uterine rupture, vasa praevia, fetal distress, or child renal failure. The median for birth weight in the cohort of all women with a pregnancy outcome after the introduction of the vaccination campaign was $3400 \mathrm{~g}$ (interquartile range $3100-3800 \mathrm{~g}$ )

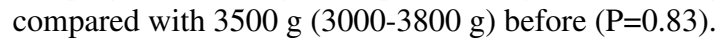

\section{Discussion}

\section{Key findings}

In 2011-12, the UK saw the largest pertussis outbreak for over a decade, with 14 deaths in 2012 in infants born before the start of the pregnancy vaccination campaign who were too young to be vaccinated themselves. The pertussis vaccination in pregnancy programme was an important public health intervention aimed at reducing morbidity and mortality in infants.

Over 700000 women in the third trimester of pregnancy were to be offered vaccination in the UK over the first year. Given relatively high background rates for several adverse outcomes during pregnancy, particularly stillbirth, it was inevitable that such events would occur in temporal association with vaccination, regardless of causality. The key challenges were to quickly identify any evidence of risks attributable to the vaccine so that any required action could be taken and, in the 
absence of such evidence, to provide robust safety data to support public health.

This study found no evidence of an increased risk of any of the extensive predefined list of adverse events related to pregnancy. In particular, there was no evidence of an increased risk of stillbirth.

\section{Strengths and limitations of the study}

Given the high uptake of the vaccine, ${ }^{21}$ the size of the database, and that vaccination was almost exclusively in primary care we were able to quickly identify a large number of vaccinated pregnant women with rapid data on outcomes. To comprehensively monitor the safety profile of the vaccination in pregnancy, optimise the use of all the available data, and overcome some of the limitations of observational studies conducted with GP data, we took several different approaches to the analysis. By comparing post-vaccination event rates from the CPRD with background data from external sources where available, we were able to consider the impact of any changes in GP recording practices. The different approaches taken to the study design add weight to the findings, with the analysis on short term risk using as much of the data as possible to maximise power, while the analyses looking at total risk after vaccination were designed to reduce confounding by accounting for changes in risk with gestational and maternal age.

We analysed data amassed six months after the start of the vaccination programme. Given that this study was designed to monitor the safety profile of the pertussis vaccine in pregnancy on a continuous basis we made no a priori calculations on the power of the study. Our results showed no significantly increased risks, and, indeed, confidence intervals are such that in general we can exclude twofold risks. The analysis presented, however, cannot rule out smaller increases in risk, and the short study period limits the possibility of examining longer term adverse events. The vaccination programme remains ongoing, and the MHRA continues to monitor the safety of the vaccine in pregnancy with the aim of identifying any smaller increases in the risk of adverse events related to pregnancy as well as longer term safety.

There is, of course, the potential for unmeasured confounding. Given the nature of this study, designed as it was to be analysed as soon as data became available to provide rapid safety data, we did not fully adjust for potential confounders. In particular, smoking, alcohol and drug use, parity, socioeconomic status, and some drug treatments are known to be associated with the risk of adverse events in pregnancy and were not adjusted for in this study. We did, however, account for two of the potentially most important confounders: maternal and gestational age.

It is possible that women choosing not to be vaccinated have inherently different risks because of unmeasured confounders such as concomitant drug treatment, medical and obstetric history, smoking, and body mass index. The sensitivity analysis including all women with a pregnancy outcome during the campaign, regardless of vaccination status, however, found no significant increases in the risk of any of the predefined adverse events related to pregnancy before and after the introduction of the vaccination campaign, suggesting that unmeasured confounding has not masked any safety signals here, assuming there have been no significant changes in the characteristics of the pregnant population.

Despite the use of constraints on the follow-up time required for patients to be eligible for analysis to try to allow for recording delay, there is still the possibility of missing event data in the CPRD. In particular, data on events in women presenting to hospital might not be recorded, meaning that we are underestimating the rate of adverse events. The severity of the events, and the fact that women experiencing them would subsequently be monitored by their GP, however, means that missing data should be minimal, though this is still a limitation of general practice electronic medical records data in general. In addition, we would not expect the level of missing data to have changed significantly within the study period so internal comparisons of event rates would remain valid. The sensitivity and specificity of the mother-child link is unknown, meaning that we could be missing some data on the child record, but again we would not expect the linkage to be substantially different between the vaccinated and historical unvaccinated cohorts.

Given that the recommendation was for vaccination in the third trimester of pregnancy, the risk of congenital malformations was not prespecified as an adverse event of interest. However, this is continuously monitored through routine

pharmacovigilance, and no signal of an increased risk has been raised. It should also be noted that no safety concerns were raised for any adverse event related to pregnancy through spontaneous suspected adverse reaction reports through the UK Yellow Card scheme (www.mhra.gov.uk/yellowcard).

We did not evaluate the effectiveness of the vaccine or the impact of the immunisation campaign on control of pertussis. Public Health England has in place a programme to evaluate these outcomes, which are important factors to balance against the evidence of safety. A further limitation of the study is that we could not evaluate factors such as optimal timing of vaccination or persistence of maternal antibody, which could influence the best timing of booster doses in future pregnancies, or the potential for transplacental antibody to interfere with the infant response to the primary immunisation schedule. These are important aspects relevant to the overall safety and effectiveness of the immunisation campaign and require further research.

\section{Implications of the study}

Pertussis remains an important disease burden in young infants, and maternal immunisation is an important public health intervention to reduce this burden. The US already recommends maternal pertussis immunisation, although to date uptake has been $\operatorname{low}^{22}$ and the evidence of safety limited. ${ }^{89}$ This is the first large controlled study of the safety of maternal pertussis immunisation. With other countries considering similar interventions, and with the early results on efficacy also coming from the UK, ${ }^{1423}$ our findings provide initial evidence for evaluating the safety of the vaccine in pregnancy for health professionals and the public and can help to inform vaccination policy making.

We thank Gayatri Amirthalingam, Nick Andrews, and Liz Miller from Public Health England for their comments on the draft manuscript.

Contributors: KD, BK, and PB devised the study and drafted and approved the manuscript. KD conducted the statistical analysis. $\mathrm{PB}$ is guarantor.

Funding: This research received no specific grant from any funding agency in the public, commercial, or not-for-profit sectors.

Competing interests: All authors have completed the ICMJE uniform disclosure form at http://www.icmje.org/coi_disclosure.pdf and declare: no support from any organisation for the submitted; no financial relationships with any organisations that might have an interest in the submitted work in the previous three years, no other relationships or activities that could appear to have influenced the submitted work. At 


\section{What is already known on this topic}

Pertussis immunisation of pregnant women has been routinely recommended in the United States since 2011 with no safety concerns arising from post-marketing surveillance

Uptake of the vaccine in the US has so far been low and evidence of safety is relatively limited

\section{What this study adds}

This study found no increased risk of stillbirth either immediately after vaccination or across the remainder of the pregnancy

There was no increased risk of maternal or neonatal death, (pre-) eclampsia, haemorrhage, fetal distress, uterine rupture, placenta or vasa praevia, caesarean delivery, low birth weight, or child renal failure

These initial data provide evidence for health professionals and the public to balance against the benefits of the vaccine and can help to inform international vaccination policy making

the time of the study, all authors were employed by the Medicines and Healthcare products Regulatory Agency (MHRA) which is an Executive Agency of the Department of Health. The MHRA has statutory responsibility to monitor the safety of medicinal products, including vaccines, on the UK market, and the study was undertaken independently of the Department of Health.

Ethical approval: The study protocol was approved by the CPRD Independent Scientific Advisory Committee. No further ethical approval was required.

Data sharing: Read code lists can be obtained from the corresponding author.

Transparency declaration: PB affirms that this manuscript is an honest, accurate, and transparent account of the study being reported; that no important aspects of the study have been omitted; and that any discrepancies from the study as planned have been explained.

1 Crowcroft NS and Pebody RG. Recent developments in pertussis. Lancet 2006:367:1926-36.

2 The complete routine immunisation schedule 2013/2014. Public Health England, 2013. www.gov.uk/government/publications/the-complete-routine-immunisation-schedule-201314. 3 NHS Immunisation Statistics, England 2011-12. NHS Information Centre for Health and Social Care, 2013. https://catalogue.ic.nhs.uk/publications/public-health/immunisation/ nhs-immu-stat-eng-2011-2012/nhs-immu-stat-eng-2011-12-rep.pdf.

4 Health Protection Agency. Laboratory-confirmed cases of pertussis reported to the enhanced pertussis surveillance programme (England and Wales). Health Protection Report 2012;6(8). www.hpa.org.uk/hpr/archives/2012/hpr0812.pdf.

5 Joint Committee on Vaccination and Immunisation. Minutes of teleconference on Thursday 30 August 2012 10.00am - 12.00am and post-teleconference discussion www.gov.uk/ government/uploads/system/uploads/attachment_data/file/223497/JCVI_minutes_Aug_ 2012 Pertussis - final.pdf.

6 Casey JR, Pichichero ME. Acellular pertussis vaccine safety in children, adolescents, and adults. Drugs 2005;65:1637-89.

7 Keital WA, Muenz LR, Decker MD, Englund JA, Mink CM, Blumberg DA, et al. A randomized controlled trial of acellular pertussis vaccines in healthy adults: dose-response comparisons of 5 vaccines and implications for booster immunization. $J$ Infect Dis 1999;180:397-403.

8 Zheteyeva YA, Moro PL, Tepper NK, Rasmussen SA, Barash FE, Revzina NV, et al. Adverse event reports after tetanus toxoid, reduced diphtheria toxoid, and acellular pertussis vaccines in pregnant women. Am J Obstet Gynecol 2012;207:59.e1-7.

9 Global Advisory Committee on Vaccine Safety. World Health Organization. Weekly Epidemiological Record 2013:29:301-12.

10 Advisory Committee on Immunization Practices. Updated recommendation for use of tetanus toxoid, reduced diphtheria toxoid and acellular pertussis vaccine (Tdap) in pregnant women and persons who have or anticipate having close contact with an infant $<12$ months. ACIP, 2011. www.cdc.gov/mmwr/preview/mmwrhtml/mm6041a4.htm.

11 Gall SA, Myers J, Pichichero M. Maternal immunization with tetanus-diphtheria-pertussis vaccine: effect on maternal and neonatal serum antibody levels. Am J Obstet Gynecol 2011;204:334.e1-5.

12 Gelfand JM, Margolis DJ, Dattani H. The UK general practice research database. In: Strom BL, ed. Pharmacoepidemiology. 4th ed. John Wiley, 2005:337-46.

13 Sammon CJ, McGrogan A, Snowball J, de Vries CS. Pandemic influenza vaccination during pregnancy: an investigation of vaccine uptake during the 2009/10 pandemic vaccination campaign in Great Britain. Hum Vaccin Immunother 2013;9:917-23.

14 Joint Committee on Vaccination and Immunisation. Minutes of the meeting on Wednesday 6 February 2013 www.gov.uk/government/uploads/system/uploads/attachment_data/ile/ 223498/JCVI minutes_February_2013 meeting - final.pdf_- final.pdf.

15 Devine S, West S, Andrews E, Tennis P, Hammad TA, Eaton S, et al. The identification of pregnancies within the general practice research database. Pharmacoepidemiol Drug Saf 2010;19:45-50.

16 Boggon R, Gallagher A, Williams T, van Staa T. Creating a mother baby link and pregnancy register for a UK population. Pharmacoepidemiol Drug Saf 2011;20:S44.

17 Gestation-specific infant mortality in England and Wales, 2010. Office for National Statistics. www.ons.gov.uk/ons/rel/child-health/gestation-specific-infant-mortality-in-england-andwales/2010/rft-1-2010.xls.

18 Infant and perinatal mortality in England and Wales by social and biological factors, 2011. Office for National Statistics. www.ons.gov.uk/ons/rel/child-health/infant-and-perinatalmortality-in-england-and-wales-by-social-and-biological-factors/2011/stb-infant-andperinatal-mortality--2011.html.

19 Tuffnell DJ, Jankowicz D, Lindow SW, Lyons G, Mason GC, Russell IF, et al. Outcomes of severe pre-eclampsia/eclampsia in Yorkshire 1999/2003. BJOG 2005;112:875-80.

20 Knight M, UKOSS. Eclampsia in the United Kingdom 2005. Br J Obstet Gynaecol 2007;114:1072-8.

21 Pertussis vaccination programme for pregnant women 2012 to 2014: monthly data by area team (England). www.gov.uk/government/uploads/system/uploads/attachment data/ file/308699/PertussisVaccineCoverage_Oct2012-Mar2014_acc2.pdf.

22 Advisory Committee on Immunization Practices. Updated recommendations for use of tetanus toxoid, reduced diphtheria toxoid, acellular pertussis vaccine (Tdap) in pregnant women. ACIP, 2012. www.cdc.gov/mmwr/preview/mmwrhtml/mm6207a4.htm.

23 Amirthalingam G, Andrews N, Campbell H, Ribeiro S, Kara E, Donegan K, et al. Effectiveness of maternal pertussis vaccination in England: an observational study. Lancet 2014 (in press).

Accepted: 18 June 2014

\section{Cite this as: BMJ 2014;349:g4219}

This is an Open Access article distributed in accordance with the Creative Commons Attribution Non Commercial (CC BY-NC 3.0) license, which permits others to distribute, remix, adapt, build upon this work non-commercially, and license their derivative works on different terms, provided the original work is properly cited and the use is non-commercial. See: http://creativecommons.org/licenses/by-nc/3.0/. 


\section{Table}

Table 1| Results of matched cohort analyses of safety of pertussis vaccination in pregnant women. Overall risk of predefined potential adverse events in vaccinated women and all women eligible for vaccination versus historical unvaccinated controls

\begin{tabular}{|c|c|c|c|c|c|c|}
\hline \multirow[b]{3}{*}{ Event ${ }^{\star}$} & \multicolumn{3}{|c|}{ Vaccinated $v$ historical unvaccinated controls } & \multicolumn{3}{|c|}{ All eligible women $v$ unvaccinated controls } \\
\hline & \multicolumn{2}{|c|}{ No (\%) events } & \multirow[b]{2}{*}{$\begin{array}{l}\text { Incidence rate ratio } \\
(95 \% \mathrm{Cl})\end{array}$} & \multicolumn{2}{|c|}{ No (\%) events } & \multirow[b]{2}{*}{$\begin{array}{l}\text { Incidence rate } \\
\text { ratio }(95 \% \mathrm{Cl})\end{array}$} \\
\hline & $\begin{array}{c}\text { Vaccinated } \\
\text { women }(n=6185)\end{array}$ & $\begin{array}{c}\text { Matched } \\
\text { unvaccinated } \\
\text { women }(n=18 \text { 523) }\end{array}$ & & $\begin{array}{c}\text { Potentially } \\
\text { vaccinated women } \\
(n=9735)\end{array}$ & $\begin{array}{c}\text { Matched } \\
\text { unvaccinated } \\
\text { women }(n=29165)\end{array}$ & \\
\hline Stillbirth & $12(0.19)$ & $42(0.23)$ & 0.85 (0.45 to 1.61$)$ & $25(0.26)$ & $61(0.21)$ & 1.21 (0.76 to 1.92$)$ \\
\hline $\begin{array}{l}\text { Neonatal death (within } 7 \\
\text { days) }\end{array}$ & $2(0.03)$ & $6(0.03)$ & $1.00(0.20$ to 4.95$)$ & $2(0.02)$ & $6(0.02)$ & 1.00 (0.20 to 4.95$)$ \\
\hline Pre-eclampsia/eclampsia & $22(0.36)$ & $54(0.29)$ & $1.22(0.74$ to 2.01$)$ & $34(0.34)$ & $196(0.67)$ & $0.52(0.36$ to 0.79$)$ \\
\hline Placenta praevia & $2(0.03)$ & $15(0.08)$ & $0.40(0.09$ to 1.75$)$ & $4(0.04)$ & $23(0.08)$ & $0.52(0.18$ to 1.51$)$ \\
\hline $\begin{array}{l}\text { Intrauterine growth } \\
\text { retardation/low birth } \\
\text { weight/weight }<2500 \mathrm{~g}\end{array}$ & $126(2.04)$ & $311(1.68)$ & $1.20(0.98$ to 1.48$)$ & $217(2.23)$ & $563(1.93)$ & 1.15 (0.98 to 1.40$)$ \\
\hline Caesarean section & $1238(20.02)$ & $3748(20.22)$ & $0.99(0.93$ to 1.06$)$ & $1879(19.30)$ & $5797(19.88)$ & $0.97(0.92$ to 1.02$)$ \\
\hline $\begin{array}{l}\text { Premature labour (without } \\
\text { delivery) }\end{array}$ & $5(0.08)$ & $21(0.11)$ & 0.71 (0.27 to 1.89$)$ & $10(0.10)$ & $16(0.05)$ & 1.88 (0.85 to 4.13$)$ \\
\hline Postpartum haemorrhage & $59(0.95)$ & $181(0.98)$ & 0.98 (0.73 to 1.31$)$ & $83(0.85)$ & $312(1.07)$ & 0.80 (0.63 to 1.01$)$ \\
\hline
\end{tabular}

${ }^{\star}$ Recorded clinical diagnosis unless specified. 